\title{
WEAK AND STRONG CONVERGENCE OF FINITE FAMILY WITH ERRORS OF NONEXPANSIVE NONSELF-MAPPINGS
}

\author{
S. PLUBTIENG AND K. UNGCHITTRAKOOL
}

Received 27 September 2005; Revised 5 May 2006; Accepted 8 May 2006

We are concerned with the study of a multistep iterative scheme with errors involving a finite family of nonexpansive nonself-mappings. We approximate the common fixed points of a finite family of nonexpansive nonself-mappings by weak and strong convergence of the scheme in a uniformly convex Banach space. Our results extend and improve some recent results, Shahzad (2005) and many others.

Copyright (c) 2006 S. Plubtieng and K. Ungchittrakool. This is an open access article distributed under the Creative Commons Attribution License, which permits unrestricted use, distribution, and reproduction in any medium, provided the original work is properly cited.

\section{Introduction}

Let $K$ be a subset of a real normed linear space $E$ and let $T$ be a self-mapping on $K$. $T$ is said to be nonexpansive provided $\|T x-T y\| \leqslant\|x-y\|$ for all $x, y \in K$.

Fixed-point iteration process for nonexpansive mappings in Banach spaces including Mann and Ishikawa iteration processes has been studied extensively by many authors to solve the nonlinear operator equations in Hilbert spaces and Banach spaces; see $[3,7,10,11,15,16]$. Tan and $\mathrm{Xu}[15]$ introduced and studied a modified Ishikawa process to approximate fixed points of nonexpansive mappings defined on nonempty closed convex bounded subsets of a uniformly convex Banach space E. Five years later, $\mathrm{Xu}$ [18] introduced iterative schemes known as Mann iterative scheme with errors and Ishikawa iterative scheme with errors. Takahashi and Tamura [14] introduced and studied a generalization of Ishikawa iterative schemes for a pair of nonexpansive mappings in Banach spaces. Recently, Khan and Fukhar-ud-din [6] extended their scheme to the modified Ishikawa iterative schemes with errors for two mappings and gave weak and strong convergence theorems. On the other hand, iterative techniques for approximating fixed points of nonexpansive nonself-mappings have been studied by various authors; see [4, 8, 13, 19]. Shahzad [12] introduced and studied an iteration scheme for 
approximating a fixed point of nonexpansive nonself-mappings (when such a fixed point exists) and gave some strong and weak convergence theorems for such mappings.

Inspired and motivated by these facts, we introduce and study a multistep iterative scheme with errors for a finite family of nonexpansive nonself-mappings. Our schemes can be viewed as an extension for two-step iterative schemes of Shahzad [12]. The scheme is defined as follows.

Let $K$ be a nonempty closed convex subset of a uniformly convex Banach space $E$, which is also a nonexpansive retract of $E$. And let $T_{1}, T_{2}, \ldots, T_{N}: K \rightarrow E$ be nonexpansive mappings, the following iteration scheme is studied:

$$
\begin{gathered}
x_{n}^{1}=P\left(\alpha_{n}^{1} T_{1} x_{n}+\beta_{n}^{1} x_{n}+\gamma_{n}^{1} u_{n}^{1}\right), \\
x_{n}^{2}=P\left(\alpha_{n}^{2} T_{2} x_{n}^{1}+\beta_{n}^{2} x_{n}+\gamma_{n}^{2} u_{n}^{2}\right), \\
\vdots \\
\vdots \\
x_{n+1}=x_{n}^{N}=P\left(\alpha_{n}^{N} T_{N} x_{n}^{N}-1+\beta_{n}^{N} x_{n}+\gamma_{n}^{N} u_{n}^{N}\right)
\end{gathered}
$$

with $x_{1} \in K, n \geqslant 1$, where $P$ is a nonexpansive retraction with respect to $K$ and $\left\{\alpha_{n}^{1}\right\}$, $\left\{\alpha_{n}^{2}\right\}, \ldots,\left\{\alpha_{n}^{N}\right\},\left\{\beta_{n}^{1}\right\},\left\{\beta_{n}^{2}\right\}, \ldots,\left\{\beta_{n}^{N}\right\},\left\{\gamma_{n}^{1}\right\},\left\{\gamma_{n}^{2}\right\}, \ldots,\left\{\gamma_{n}^{N}\right\}$ are sequences in $[0,1]$ with $\alpha_{n}^{i}+$ $\beta_{n}^{i}+\gamma_{n}^{i}=1$ for all $i=1,2, \ldots, N$, and $\left\{u_{n}^{1}\right\},\left\{u_{n}^{2}\right\}, \ldots,\left\{u_{n}^{N}\right\}$ are bounded sequences in $K$.

For $N=2, T_{1}=T_{2} \equiv T, \beta_{n}=\alpha_{n}^{1}, \alpha_{n}=\alpha_{n}^{2}$, and $\gamma_{n}^{1}=\gamma_{n}^{2} \equiv 0$, then (1.1) reduces to the scheme for a mapping defined by Shahzad [12]:

$$
\begin{aligned}
x_{1} & =x \in K, \\
x_{n+1} & =P\left(\left(1-\alpha_{n}\right) x_{n}+\alpha_{n} T P\left[\left(1-\beta_{n}\right) x_{n}+\beta_{n} T x_{n}\right]\right),
\end{aligned}
$$

where $\left\{\alpha_{n}\right\},\left\{\beta_{n}\right\}$ are sequences in $[0,1]$.

For $N=2, T_{1}, T_{2}: K \rightarrow K, T_{1}=T, T_{2}=S$, and $y_{n}=x_{n}^{1}$, then (1.1) reduces to the scheme with errors for two mappings defined by

$$
\begin{gathered}
x_{1}=x \in K, \\
y_{n}=\alpha_{n}^{1} T x_{n}+\beta_{n}^{1} x_{n}+\gamma_{n}^{1} u_{n}^{1}, \\
x_{n+1}=x_{n}^{2}=\alpha_{n}^{2} S y_{n}+\beta_{n}^{2} x_{n}+\gamma_{n}^{2} u_{n}^{2},
\end{gathered}
$$

where $\left\{\alpha_{n}^{1}\right\},\left\{\alpha_{n}^{2}\right\},\left\{\beta_{n}^{1}\right\},\left\{\beta_{n}^{2}\right\},\left\{\gamma_{n}^{1}\right\},\left\{\gamma_{n}^{2}\right\}$ are sequences in $[0,1]$ with $\alpha_{n}^{1}+\beta_{n}^{1}+\gamma_{n}^{1}=1=\alpha_{n}^{2}+$ $\beta_{n}^{2}+\gamma_{n}^{2}$ and $\left\{u_{n}^{1}\right\},\left\{u_{n}^{2}\right\}$ are bounded sequences in $K$.

It is our purpose in this paper to establish several weak and strong convergence theorems of the multistep iterative scheme with errors for a finite family of nonexpansive nonself-mappings. More precisely, we prove weak convergence of these implicit iteration 
processes in a uniformly convex Banach space which has the Kadec-Klee property. The results presented in this paper extend and improve the corresponding ones announced by Shahzad [12], and many others.

\section{Preliminaries}

In this section, we recall the well-known concepts and results.

Let $E$ be a real Banach space. A subset $K$ of $E$ is said to be a retract of $E$ if there exists a continuous map $P: E \rightarrow K$ such that $P x=x$ for all $x \in K$. A map $P: E \rightarrow E$ is said to be a retraction if $P^{2}=P$. It follows that if a map $P$ is a retraction, then $P y=y$ for all $y$ in the range of $P$. A mapping $T: K \rightarrow E$ is called demiclosed with respect to $y \in E$ if for each sequence $\left\{x_{n}\right\}$ in $K$ and each $x \in E, x_{n} \rightarrow x$ and $T x_{n} \rightarrow y$ imply that $x \in K$ and $T x=y$. A Banach space $E$ is said to satisfy Opial's condition [9] if for any sequence $\left\{x_{n}\right\}$ in $E, x_{n} \rightarrow x$ implies that

$$
\underset{n \rightarrow \infty}{\limsup }\left\|x_{n}-x\right\|<\limsup _{n \rightarrow \infty}\left\|x_{n}-y\right\|
$$

for all $y \in E$ with $x \neq y$. A Banach space $E$ is said to have the Kadec-Klee property if for every sequence $\left\{x_{n}\right\}$ in $E, x_{n} \rightarrow x$ and $\left\|x_{n}\right\| \rightarrow\|x\|$ together imply $\left\|x_{n}-x\right\| \rightarrow 0$. A family $\left\{T_{i}: i=1,2, \ldots, N\right\}$ of $N$ nonself-mappings of $K$ (i.e., $T_{i}: K \rightarrow E$ ) with $F=\bigcap_{i=1}^{N} F\left(T_{i}\right) \neq \varnothing$ is said to satisfy condition $(B)$ on $K$ if there is a nondecreasing function $f:[0, \infty) \rightarrow[0, \infty)$ with $f(0)=0$ and $f(r)>0$ for all $r \in(0, \infty)$ such that for all $x \in K$,

$$
\max _{1 \leqslant i \leqslant N}\left\{\left\|x-T_{i} x\right\|\right\} \geqslant f(d(x, F)) .
$$

The family $\left\{T_{i}: i=1,2, \ldots, N\right\}$ is said to satisfy condition $\left(A^{N}\right)$ if $(2.2)$ is replaced by $1 / N \sum_{i=1}^{N}\left\|x-T_{i} x\right\| \geqslant f(d(x, F))$ for all $x \in K$. Note that condition $(B)$ reduces to condition $\left(A^{N}\right)$ when $\left\|x-T_{1} x\right\|=\left\|x-T_{2} x\right\|=\cdots=\left\|x-T_{N} x\right\|$.

A mapping $T: K \rightarrow E$ is called semicompact if any sequence $\left\{x_{n}\right\}$ in $K$ satisfying $\| x_{n}-$ $T x_{n} \| \rightarrow 0$ as $n \rightarrow \infty$ has a convergent subsequence.

Lemma 2.1 (Tan and $\mathrm{Xu}[15]$ ). Let $\left\{s_{n}\right\},\left\{t_{n}\right\}$ be two nonnegative sequences satisfying

$$
s_{n+1} \leqslant s_{n}+t_{n}, \quad \forall n \geqslant 1 .
$$

If $\sum_{n=1}^{\infty} t_{n}<\infty$, then $\lim _{n \rightarrow \infty} s_{n}$ exists. Moreover, if there exists a subsequence $\left\{s_{n_{j}}\right\}$ of $\left\{s_{n}\right\}$ such that $s_{n_{j}} \rightarrow 0$ as $j \rightarrow \infty$, then $s_{n} \rightarrow 0$ as $n \rightarrow \infty$.

Lemma 2.2 (Xu [17]). Let $p>1$ and $R>0$ be two fixed numbers and $E$ a Banach space. Then $E$ is uniformly convex if and only if there exists a continuous, strictly increasing, and convex function $g:[0, \infty) \rightarrow[0, \infty)$ with $g(0)=0$ such that $\|\lambda x+(1-\lambda) y\|^{p} \leqslant \lambda\|x\|^{p}+$ $(1-\lambda)\|y\|^{p}-W_{p}(\lambda) g(\|x-y\|)$ for all $x, y \in B_{R}(0)=\{x \in E:\|x\| \leqslant R\}$, and $\lambda \in[0,1]$, where $W_{p}(\lambda)=\lambda(1-\lambda)^{p}+\lambda^{p}(1-\lambda)$.

Lemma 2.3 (Kaczor [5]). Let E be a real reflexive Banach space such that its dual $E^{*}$ has the Kadec-Klee property. Let $\left\{x_{n}\right\}$ be a bounded sequence in $E$ and $x^{*}, y^{*} \in \omega_{w}\left(x_{n}\right)$; here $\omega_{w}\left(x_{n}\right)$ 
denote the set of all weak subsequential limits of $\left\{x_{n}\right\}$. Suppose $\lim _{n \rightarrow \infty}\left\|t x_{n}+(1-t) x^{*}-y^{*}\right\|$ exists for all $t \in[0,1]$. Then $x^{*}=y^{*}$.

Lemma 2.4 (Browder [1]). Let E be a uniformly convex Banach space, K a nonempty closed convex subset of $E$, and $T: K \rightarrow E$ a nonexpansive mapping. Then $I-T$ is demiclosed at zero.

\section{Main results}

In this section, we prove weak and strong convergence theorems of the iterative scheme given in (1.1) for a finite family of nonexpansive mappings in a Banach space. In order to prove our main results, the following lemmas are needed.

Lemma 3.1. Let $E$ be a uniformly convex Banach space and $K$ a nonempty closed convex subset of $E$ which is also a nonexpansive retract of $E$. Let $T_{1}, T_{2}, \ldots, T_{N}: K \rightarrow E$ be nonexpansive mappings. Let $\left\{x_{n}\right\}$ be the sequence defined by (1.1) with $\sum_{n=1}^{\infty} \gamma_{n}^{i}<\infty$ for each $i=1,2, \ldots, N$. If $\bigcap_{i=1}^{N} F\left(T_{i}\right) \neq \varnothing$, then $\lim _{n \rightarrow \infty}\left\|x_{n}-x^{*}\right\|$ exists for all $x^{*} \in \bigcap_{i=1}^{N} F\left(T_{i}\right)$.

Proof. For each $n \geqslant 1$, we note that

$$
\begin{aligned}
\left\|x_{n}^{1}-x^{*}\right\| & \leqslant \alpha_{n}^{1}\left\|T_{1} x_{n}-x^{*}\right\|+\beta_{n}^{1}\left\|x_{n}-x^{*}\right\|+\gamma_{n}^{1}\left\|u_{n}^{1}-x^{*}\right\| \\
& \leqslant \alpha_{n}^{1}\left\|x_{n}-x^{*}\right\|+\beta_{n}^{1}\left\|x_{n}-x^{*}\right\|+\gamma_{n}^{1}\left\|u_{n}^{1}-x^{*}\right\| \\
& \leqslant\left\|x_{n}-x^{*}\right\|+d_{n}^{0},
\end{aligned}
$$

where $d_{n}^{0}=\gamma_{n}^{1}\left\|u_{n}^{1}-x^{*}\right\|$. Since $\sum_{n=1}^{\infty} \gamma_{n}^{1}<\infty, \sum_{n=1}^{\infty} d_{n}^{0}<\infty$. Next, we note that

$$
\begin{aligned}
\left\|x_{n}^{2}-x^{*}\right\| & \leqslant \alpha_{n}^{2}\left\|T_{2} x_{n}^{1}-x^{*}\right\|+\beta_{n}^{2}\left\|x_{n}-x^{*}\right\|+\gamma_{n}^{2}\left\|u_{n}^{2}-x^{*}\right\| \\
& \leqslant \alpha_{n}^{2}\left\|x_{n}^{1}-x^{*}\right\|+\beta_{n}^{2}\left\|x_{n}-x^{*}\right\|+\gamma_{n}^{2}\left\|u_{n}^{2}-x^{*}\right\| \\
& =\left(\alpha_{n}^{2}+\beta_{n}^{2}\right)\left\|x_{n}-x^{*}\right\|+\alpha_{n}^{2} d_{n}^{0}+\gamma_{n}^{2}\left\|u_{n}^{2}-x^{*}\right\| \\
& \leqslant\left\|x_{n}-x^{*}\right\|+d_{n}^{1},
\end{aligned}
$$

where $d_{n}^{1}=\alpha_{n}^{2} d_{n}^{0}+\gamma_{n}^{2}\left\|u_{n}^{2}-x^{*}\right\|$. Since $\sum_{n=1}^{\infty} d_{n}^{0}<\infty$ and $\sum_{n=1}^{\infty} \gamma_{n}^{2}<\infty, \sum_{n=1}^{\infty} d_{n}^{1}<\infty$. Similarly, we have

$$
\begin{aligned}
\left\|x_{n}^{3}-x^{*}\right\| & \leqslant \alpha_{n}^{3}\left\|x_{n}^{2}-x^{*}\right\|+\beta_{n}^{3}\left\|x_{n}-x^{*}\right\|+\gamma_{n}^{3}\left\|u_{n}^{3}-x^{*}\right\| \\
& \leqslant \alpha_{n}^{3}\left[|| x_{n}-x^{*} \|+d_{n}^{1}\right]+\beta_{n}^{3}\left\|x_{n}-x^{*}\right\|+\gamma_{n}^{3}\left\|u_{n}^{3}-x^{*}\right\| \\
& \leqslant\left\|x_{n}-x^{*}\right\|+\alpha_{n}^{3} d_{n}^{1}+\gamma_{n}^{3}\left\|u_{n}^{3}-x^{*}\right\|=\left\|x_{n}-x^{*}\right\|+d_{n}^{2},
\end{aligned}
$$

where $d_{n}^{2}=\alpha_{n}^{3} d_{n}^{1}+\gamma_{n}^{3}\left\|u_{n}^{3}-x^{*}\right\|$, so $\sum_{n=1}^{\infty} d_{n}^{2}<\infty$. 
By continuing the above method, there exists a nonnegative real sequence $\left\{d_{n}^{i-1}\right\}$ such that $\sum_{n=1}^{\infty} d_{n}^{i-1}<\infty$ and

$$
\left\|x_{n}^{i}-x^{*}\right\| \leqslant\left\|x_{n}-x^{*}\right\|+d_{n}^{i-1}, \quad \forall n \geqslant 1, \forall i=1,2, \ldots, N .
$$

Thus $\left\|x_{n+1}-x^{*}\right\|=\left\|x_{n}^{N}-x^{*}\right\| \leqslant\left\|x_{n}-x^{*}\right\|+d_{n}^{N-1}$ for all $n \in N$. Hence, by Lemma 2.1, $\lim _{n \rightarrow \infty}\left\|x_{n}-x^{*}\right\|$ exists. This completes the proof.

Lemma 3.2. Let $E$ be a uniformly convex Banach space and $K$ a nonempty closed convex subset of $E$ which is also a nonexpansive retract of $E$. Let $T_{1}, T_{2}, \ldots, T_{N}: K \rightarrow E$ be nonexpansive mappings. Let $\left\{x_{n}\right\}$ be the sequence defined by (1.1) with $\sum_{n=1}^{\infty} \gamma_{n}^{i}<\infty$ and $\left\{\alpha_{n}^{i}\right\} \subseteq$ $[\varepsilon, 1-\varepsilon]$ for all $i=1,2, \ldots, N$, for some $\varepsilon \in(0,1)$. If $\bigcap_{i=1}^{N} F\left(T_{i}\right) \neq \varnothing$, then $\lim _{n \rightarrow \infty} \| x_{n}-$ $T_{i} x_{n} \|=0$ for all $i=1,2, \ldots, N$.

Proof. Let $x^{*} \in \bigcap_{i=1}^{N} F\left(T_{i}\right)$. Then, by Lemma 3.1, $\lim _{n \rightarrow \infty}\left\|x_{n}-x^{*}\right\|$ exists. Let $\lim _{n \rightarrow \infty} \|$ $x_{n}-x^{*} \|=r$. If $r=0$, then by the continuity of each $T_{i}$ the conclusion follows. Suppose that $r>0$. Firstly, we are now to show that $\lim _{n \rightarrow \infty}\left\|T_{N} x_{n}-x_{n}\right\|=0$. Since $\left\{x_{n}\right\}$ and $\left\{u_{n}^{i}\right\}$ are bounded for all $i=1,2, \ldots, N$, there exists $R>0$ such that $x_{n}-x^{*}+\gamma_{n}^{i}\left(u_{n}^{i}-x_{n}\right)$, $T_{i} x_{n}^{i-1}-x^{*}+\gamma_{n}^{i}\left(u_{n}^{i}-x_{n}\right) \in B_{R}(0)$ for all $n \geqslant 1$ and for all $i=1,2, \ldots, N$. Using Lemma 2.2, we have

$$
\begin{aligned}
\left\|x_{n}^{N}-x^{*}\right\|^{2} \leqslant & \left\|\alpha_{n}^{N} T_{N} x_{n}^{N-1}+\beta_{n}^{N} x_{n}+\gamma_{n}^{N} u_{n}^{N}-x^{*}\right\|^{2} \\
= & \left\|\alpha_{n}^{N}\left(T_{N} x_{n}^{N-1}-x^{*}+\gamma_{n}^{N}\left(u_{n}^{N}-x_{n}\right)\right)+\left(1-\alpha_{n}^{N}\right)\left(x_{n}-x^{*}+\gamma_{n}^{N}\left(u_{n}^{N}-x_{n}\right)\right)\right\|^{2} \\
\leqslant & \alpha_{n}^{N}\left\|T_{N} x_{n}^{N-1}-x^{*}+\gamma_{n}^{N}\left(u_{n}^{N}-x_{n}\right)\right\|^{2}+\left(1-\alpha_{n}^{N}\right)\left\|x_{n}-x^{*}+\gamma_{n}^{N}\left(u_{n}^{N}-x_{n}\right)\right\|^{2} \\
& -W_{2}\left(\alpha_{n}^{N}\right) g\left(\left\|T_{N} x_{n}^{N-1}-x_{n}\right\|\right) \\
\leqslant & \alpha_{n}^{N}\left(\left\|x_{n}^{N-1}-x^{*}\right\|+\gamma_{n}^{N}\left\|u_{n}^{N}-x_{n}\right\|\right)^{2}+\left(1-\alpha_{n}^{N}\right)\left(\left\|x_{n}-x^{*}\right\|+\gamma_{n}^{N}\left\|u_{n}^{N}-x_{n}\right\|\right)^{2} \\
& -W_{2}\left(\alpha_{n}^{N}\right) g\left(\left\|T_{N} x_{n}^{N-1}-x_{n}\right\|\right) \\
\leqslant & \alpha_{n}^{N}\left(\left\|x_{n}-x^{*}\right\|+d_{n}^{N-2}+\gamma_{n}^{N}\left\|u_{n}^{N}-x_{n}\right\|\right)^{2} \\
& +\left(1-\alpha_{n}^{N}\right)\left(\left\|x_{n}-x^{*}\right\|+d_{n}^{N-2}+\gamma_{n}^{N}\left\|u_{n}^{N}-x_{n}\right\|\right)^{2} \\
& -W_{2}\left(\alpha_{n}^{N}\right) g\left(\left\|T_{N} x_{n}^{N-1}-x_{n}\right\|\right) \\
= & \left(\left\|x_{n}-x^{*}\right\|+\lambda_{n}^{N-2}\right)^{2}-W_{2}\left(\alpha_{n}^{N}\right) g\left(\left\|T_{N} x_{n}^{N-1}-x_{n}\right\|\right),
\end{aligned}
$$

where $\lambda_{n}^{N-2}:=d_{n}^{N-2}+\gamma_{n}^{N}\left\|u_{n}^{N}-x^{*}\right\|$. Observe that $\varepsilon^{3} \leqslant W_{2}\left(\alpha_{n}^{N}\right)$ now (3.5) implies that $\varepsilon^{3} g\left(\left\|T_{N} x_{n}^{N-1}-x_{n}\right\|\right) \leqslant\left\|x_{n}-x^{*}\right\|^{2}-\left\|x_{n+1}-x^{*}\right\|^{2}+\rho_{n}^{N-2}$, where $\rho_{n}^{N-2}:=2 \lambda_{n}^{N-2} \| x_{n}-$ $x^{*} \|^{2}+\left(\lambda_{n}^{N-2}\right)^{2}$. Since $\sum_{n=1}^{\infty} d_{n}^{N-2}<\infty$ and $\sum_{n=1}^{\infty} \gamma_{n}^{N-2}<\infty$, we get $\sum_{n=1}^{\infty} \rho_{n}^{N-2}<\infty$. This implies that $\lim _{n \rightarrow \infty} g\left(\left\|T_{N} x_{n}^{N-1}-x_{n}\right\|\right)=0$. Since $g$ is strictly increasing and continuous 
at 0 , it follows that $\lim _{n \rightarrow \infty}\left\|T_{N} x_{n}^{N-1}-x_{n}\right\|=0$. Note that

$$
\begin{aligned}
\left\|x_{n}-x^{*}\right\| & \leqslant\left\|x_{n}-T_{N} x_{n}^{N-1}\right\|+\left\|T_{N} x_{n}^{N-1}-x^{*}\right\| \\
& \leqslant\left\|x_{n}-T_{N} x_{n}^{N-1}\right\|+\left\|x_{n}^{N-1}-x^{*}\right\|,
\end{aligned}
$$

for all $n \geqslant 1$. Thus $r=\lim _{n \rightarrow \infty}\left\|x_{n}-x^{*}\right\| \leqslant \liminf _{n \rightarrow \infty}\left\|x_{n}^{N-1}-x^{*}\right\| \leqslant \limsup _{n \rightarrow \infty} \| x_{n}^{N-1}-$ $x^{*} \| \leqslant r$ and therefore $\lim _{n \rightarrow \infty}\left\|x_{n}^{N-1}-x^{*}\right\|=r$. Using the same argument in the proof above, we have

$$
\begin{aligned}
\left\|x_{n}^{N-1}-x^{*}\right\|^{2} \leqslant & \alpha_{n}^{N-1}\left(\left\|x_{n}^{N-2}-x^{*}\right\|+\gamma_{n}^{N-1}\left\|u_{n}^{N-1}-x^{*}\right\|\right)^{2} \\
& +\left(1-\alpha_{n}^{N-1}\right)\left(\left\|x_{n}-x^{*}\right\|+\gamma_{n}^{N-1}\left\|u_{n}^{N-1}-x^{*}\right\|\right)^{2} \\
& -W_{2}\left(\alpha_{n}^{N-1}\right) g\left(\left\|T_{N-1} x_{n}^{N-2}-x_{n}\right\|\right) \\
\leqslant & \alpha_{n}^{N-1}\left(\left\|x_{n}-x^{*}\right\|+d_{n}^{N-3}+\gamma_{n}^{N-1}\left\|u_{n}^{N-1}-x^{*}\right\|\right)^{2} \\
& +\left(1-\alpha_{n}^{N-1}\right)\left(\left\|x_{n}-x^{*}\right\|+d_{n}^{N-3}+\gamma_{n}^{N-1}\left\|u_{n}^{N-1}-x^{*}\right\|\right)^{2} \\
& -W_{2}\left(\alpha_{n}^{N-1}\right) g\left(\left\|T_{N-1} x_{n}^{N-2}-x_{n}\right\|\right) \\
\leqslant & \left\|x_{n}-x^{*}\right\|^{2}+\rho_{n}^{N-3}-W_{2}\left(\alpha_{n}^{N-1}\right) g\left(\left\|T_{N-1} x_{n}^{N-2}-x_{n}\right\|\right) .
\end{aligned}
$$

This implies that $\varepsilon^{3} g\left(\left\|T_{N-1} x_{n}^{N-2}-x_{n}\right\|\right) \leqslant\left\|x_{n}-x^{*}\right\|^{2}-\left\|x_{n}^{N-1}-x^{*}\right\|^{2}+\rho_{n}^{N-3}$ and therefore $\lim _{n \rightarrow \infty}\left\|T_{N-1} x_{n}^{N-2}-x_{n}\right\|=0$. Thus, we have

$$
\begin{aligned}
\left\|x_{n}-T_{N} x_{n}\right\| & \leqslant\left\|x_{n}-T_{N} x_{n}^{N-1}\right\|+\left\|T_{N} x_{n}^{N-1}-T_{N} x_{n}\right\| \\
& \leqslant\left\|x_{n}-T_{N} x_{n}^{N-1}\right\|+\left\|x_{n}^{N-1}-x_{n}\right\| \\
& =\left\|x_{n}-T_{N} x_{n}^{N-1}\right\|+\left\|P\left(\alpha_{n}^{N-1} T_{N-1} x_{n}^{N-2}+\beta_{n}^{N-1} x_{n}+\gamma_{n}^{N-1} u_{n}^{N-1}\right)-P x_{n}\right\| \\
& \leqslant\left\|x_{n}-T_{N} x_{n}^{N-1}\right\|+\alpha_{n}^{N-1}\left\|x_{n}-T_{N-1} x_{n}^{N-2}\right\|+\gamma_{n}^{N-1}\left\|u_{n}^{N-1}-x_{n}\right\| .
\end{aligned}
$$

Since $\lim _{n \rightarrow \infty}\left\|x_{n}-T_{N} x_{n}^{N-1}\right\|=0, \lim _{n \rightarrow \infty}\left\|x_{n}-T_{N-1} x_{n}^{N-2}\right\|=0$, and $\sum_{n=1}^{\infty} \gamma_{n}^{N-1}<\infty$, it follows that $\lim _{n \rightarrow \infty}\left\|x_{n}-T_{N} x_{n}\right\|=0$. Similarly, by using the same argument as in the proof above, we have $\lim _{n \rightarrow \infty}\left\|x_{n}-T_{N-2} x_{n}^{N-3}\right\|=\lim _{n \rightarrow \infty}\left\|x_{n}-T_{N-3} x_{n}^{N-4}\right\|=, \ldots,=$ $\lim _{n \rightarrow \infty}\left\|x_{n}-T_{2} x_{n}^{1}\right\|=0$. This implies that $\lim _{n \rightarrow \infty}\left\|x_{n}-T_{N-1} x_{n}\right\|=\lim _{n \rightarrow \infty}\left\|x_{n}-T_{N-2} x_{n}\right\|=$ $, \ldots,=\lim _{n \rightarrow \infty}\left\|x_{n}-T_{3} x_{n}\right\|=0$. It remains to show that

$$
\lim _{n \rightarrow \infty}\left\|x_{n}-T_{1} x_{n}\right\|=0, \quad \lim _{n \rightarrow \infty}\left\|x_{n}-T_{2} x_{n}\right\|=0
$$


Note that

$$
\begin{aligned}
\left\|x_{n}^{1}-x^{*}\right\|^{2} \leqslant & \alpha_{n}^{1}\left(\left\|x_{n}-x^{*}\right\|+\gamma_{n}^{1}\left\|u_{n}^{1}-x^{*}\right\|\right)^{2} \\
& +\left(1-\alpha_{n}^{1}\right)\left(\left\|x_{n}-x^{*}\right\|+\gamma_{n}^{1}\left\|u_{n}^{1}-x^{*}\right\|\right)^{2}-W_{2}\left(\alpha_{n}^{1}\right) g\left(\left\|T_{1} x_{n}-x_{n}\right\|\right) \\
= & \left(\left\|x_{n}-x^{*}\right\|+\gamma_{n}^{1}\left\|u_{n}^{1}-x^{*}\right\|\right)^{2}-W_{2}\left(\alpha_{n}^{1}\right) g\left(\left\|T_{1} x_{n}-x_{n}\right\|\right) .
\end{aligned}
$$

Thus, we have $\varepsilon^{3} g\left(\left\|T_{1} x_{n}-x_{n}\right\|\right) \leqslant\left(\left\|x_{n}-x^{*}\right\|+\gamma_{n}^{1}\left\|u_{n}^{1}-x^{*}\right\|\right)^{2}-\left\|x_{n}^{1}-x^{*}\right\|^{2}$ and therefore $\lim _{n \rightarrow \infty}\left\|T_{1} x_{n}-x_{n}\right\|=0$. Since $\left\|x_{n}-T_{2} x_{n}\right\| \leqslant\left\|x_{n}-T_{2} x_{n}^{1}\right\|+\alpha_{n}^{1}\left\|T_{1} x_{n}-x_{n}\right\|+\gamma_{n}^{1} \| u_{n}^{1}-$ $x_{n} \|$, it implies that $\lim _{n \rightarrow \infty}\left\|T_{2} x_{n}-x_{n}\right\|=0$. Therefore $\lim _{n \rightarrow \infty}\left\|T_{i} x_{n}-x_{n}\right\|=0$ for all $i=1,2, \ldots, N$.

Theorem 3.3. Let $E$ be a uniformly convex Banach space and let $K$ be a nonempty closed convex subset of $E$ which is also a nonexpansive retract of $E$. Let $T_{1}, T_{2}, \ldots, T_{N}: K \rightarrow E$ be nonexpansive mappings which are satisfying condition $(B)$. Let $\left\{x_{n}\right\}$ be the sequence defined by (1.1) with $\sum_{n=1}^{\infty} \gamma_{n}^{i}<\infty$ and $\left\{\alpha_{n}^{i}\right\} \subseteq[\varepsilon, 1-\varepsilon]$ for all $i=1,2, \ldots, N$ for some $\varepsilon \in(0,1)$. If $F:=\bigcap_{i=1}^{N} F\left(T_{i}\right) \neq \varnothing$, then $\left\{x_{n}\right\}$ converges strongly to a common fixed point in $F$.

Proof. By Lemma 3.2, $\lim _{n \rightarrow \infty}\left\|T_{i} x_{n}-x_{n}\right\|=0$ for all $i=1,2, \ldots, N$. Now by condition $(B)$, $f\left(d\left(x_{n}, F\right)\right) \leqslant M_{n}:=\max _{1 \leqslant i \leqslant N}\left\{\left\|T_{i} x_{n}-x_{n}\right\|\right\}$ for all $n \in \mathbb{N}$. Hence $\lim _{n \rightarrow \infty} f\left(d\left(x_{n}, F\right)\right)=$ 0 . Since $f$ is a nondecreasing function and $f(0)=0$, therefore $\lim _{n \rightarrow \infty} d\left(x_{n}, F\right)=0$.

Now we can choose a subsequence $\left\{x_{n_{j}}\right\}$ of $\left\{x_{n}\right\}$ and a sequence $\left\{y_{j}\right\} \in F$ such that $\left\|x_{n_{j}}-y_{j}\right\|<2^{-j}$. By the following method of the proof of Tan and Xu [15], we get that $\left\{y_{j}\right\}$ is a Cauchy sequence in $F$ and so it converges. Let $y_{j} \rightarrow y$. Since $F$ is closed, therefore $y \in F$ and then $x_{n_{j}} \rightarrow y$. By Lemma 3.1, $\lim _{n \rightarrow \infty}\left\|x_{n}-x^{*}\right\|$ exists for all $x^{*} \in F, x_{n} \rightarrow y \in$ $F$.

For $N=2, T_{1}=T_{2} \equiv T, \beta_{n}=\alpha_{n}^{1}, \alpha_{n}=\alpha_{n}^{2}$, and $\gamma_{n}^{1}=\gamma_{n}^{2} \equiv 0$ in Theorem 3.3, we obtain the following results.

Corollary 3.4 (see [12, Theorem 3.6]). Let E be a real uniformly convex Banach space and $K$ a nonempty closed convex subset of $E$ which is also a nonexpansive retract of $E$. Let $T: K \rightarrow E$ be a nonexpansive mapping with $F(T) \neq \varnothing$. Let $\left\{\alpha_{n}\right\}$ and $\left\{\beta_{n}\right\}$ be sequences in $[\varepsilon, 1-\varepsilon]$ for some $\varepsilon \in(0,1)$. From arbitrary $x_{1} \in K$, define the sequence $\left\{x_{n}\right\}$ by the recursion (1.2). Suppose T satisfies condition $\left(A^{1}\right)$. Then $\left\{x_{n}\right\}$ converges strongly to some fixed point of $T$.

When $N=2, S=T_{1}, T=T_{2}: C \rightarrow C$, and $y_{n}=x_{n}^{1}$ in Theorem 3.3, we obtain strong convergence theorem as follows.

Corollary 3.5. Let $E$ be a uniformly convex Banach space and let $C$ be a nonempty closed convex subset of $E$ which is also a nonexpansive retract of $E$. Let $S, T$ be nonexpansive mappings of $C$ into itself satisfying condition $\left(A^{2}\right)$, and let $\left\{x_{n}\right\}$ be sequence defined by (1.3) with $\sum_{n=1}^{\infty} \gamma_{n}^{1}<\infty, \sum_{n=1}^{\infty} \gamma_{n}^{2}<\infty$ and $0<\delta \leqslant \alpha_{n}^{1}, \alpha_{n}^{2} \leqslant 1-\delta<1$ for all $n \in \mathbb{N}$. If $F:=F(S) \cap F(T) \neq \varnothing$, then $\left\{x_{n}\right\}$ converges strongly to a common fixed point of $S$ and $T$. 
Theorem 3.6. Let $E$ be a uniformly convex Banach space and let $K$ be a nonempty closed convex subset of $E$ which is also a nonexpansive retract of $E$. Let $T_{1}, T_{2}, \ldots, T_{N}: K \rightarrow E$ be nonexpansive mappings. Suppose that one of the mappings in $\left\{T_{i}: i=1,2, \ldots, N\right\}$ is semicompact. Let $\left\{x_{n}\right\}$ be the sequence defined by (1.1) with $\sum_{n=1}^{\infty} \gamma_{n}^{i}<\infty$ and $\left\{\alpha_{n}^{i}\right\} \subseteq[\varepsilon, 1-\varepsilon]$ for all $i=1,2, \ldots, N$ for some $\varepsilon \in(0,1)$. If $F:=\bigcap_{i=1}^{N} F\left(T_{i}\right) \neq \varnothing$, then $\left\{x_{n}\right\}$ converges strongly to a common fixed point in $F$.

Proof. Suppose that $T_{i_{0}}$ is semicompact for some $i_{0}=1,2, \ldots, N$. By Lemma 3.1, we have $\lim _{n \rightarrow \infty}\left\|x_{n}-T_{i_{0}} x_{n}\right\|=0$. So there exists a subsequence $\left\{x_{n_{j}}\right\}$ of $\left\{x_{n}\right\}$ such that $x_{n_{j}} \rightarrow$ $x^{*} \in K$ as $j \rightarrow \infty$. Now Lemma 3.2 guarantees that $\lim _{j \rightarrow \infty}\left\|x_{n_{j}}-T_{l} x_{n_{j}}\right\|=0$ for all $l=$ $1,2, \ldots, N$ and so $\left\|x^{*}-T_{l} x^{*}\right\|=0$ for all $l=1,2, \ldots, N$. This implies that $x^{*} \in F$. By Lemma 3.1, $\lim _{n \rightarrow \infty}\left\|x_{n}-x^{*}\right\|$ exists and then $\lim _{n \rightarrow \infty}\left\|x_{n}-x^{*}\right\|=\lim _{j \rightarrow \infty}\left\|x_{n_{j}}-x^{*}\right\|=0$. This completes the proof.

Theorem 3.7. Let E be a uniformly convex Banach space satisfying the Opial's condition and $K$ a nonempty closed convex subset of $E$ which is also a nonexpansive retract of $E$. Let $T_{1}, T_{2}, \ldots, T_{N}: K \rightarrow E$ be nonexpansive mappings and let $\left\{x_{n}\right\}$ be a sequence defined by (1.1) with $\sum_{n=1}^{\infty} \gamma_{n}^{i}<\infty$ and $\left\{\alpha_{n}^{i}\right\} \subseteq[\varepsilon, 1-\varepsilon]$ for all $i=1,2, \ldots, N$ for some $\varepsilon \in(0,1)$. If $F:=$ $\bigcap_{i=1}^{N} F\left(T_{i}\right) \neq \varnothing$, then $\left\{x_{n}\right\}$ converges weakly to a common fixed point in $F$.

Proof. Let $x^{*} \in F$. Then as proved in Lemma 3.1, $\lim _{x \rightarrow \infty}\left\|x_{n}-x^{*}\right\|$ exists. Now we prove that $\left\{x_{n}\right\}$ has a unique weak subsequential limit in $F$. To prove this, let $x_{n_{i}} \rightarrow z_{1}$ and $x_{n_{j}} \rightarrow z_{2}$ for some subsequences $\left\{x_{n_{i}}\right\},\left\{x_{n_{j}}\right\}$ of $\left\{x_{n}\right\}$. By Lemma 3.2,

$$
\lim _{i \rightarrow \infty}\left\|x_{n_{i}}-T_{k} x_{n_{i}}\right\|=0=\lim _{j \rightarrow \infty}\left\|x_{n_{j}}-T_{k} x_{n_{j}}\right\|
$$

for all $k=1,2, \ldots, N$ and by Lemma 2.4 insures that $I-T_{k}$ are demiclosed at zero for all $k=1,2, \ldots, N$. Therefore we obtain $T_{k} z_{1}=z_{1}$ and $T_{k} z_{2}=z_{2}$ for all $k=1,2, \ldots, N$. Then $z_{1}, z_{2} \in F$. Next, we prove the uniqueness. Suppose that $z_{1} \neq z_{2}$, then by the Opial's condition $\lim _{n \rightarrow \infty}\left\|x_{n}-z_{1}\right\|=\lim _{i \rightarrow \infty}\left\|x_{n_{i}}-z_{1}\right\|<\lim _{i \rightarrow \infty}\left\|x_{n_{i}}-z_{2}\right\|=\lim _{j \rightarrow \infty}\left\|x_{n_{j}}-z_{2}\right\|<$ $\lim _{j \rightarrow \infty}\left\|x_{n_{j}}-z_{1}\right\|=\lim _{n \rightarrow \infty}\left\|x_{n}-z_{1}\right\|$. This is a contradiction. Hence $\left\{x_{n}\right\}$ converges weakly to a point in $F$.

LEMma 3.8. Let $E$ be a real uniformly convex Banach space and $K$ a nonempty closed convex subset of $E$ which is also a nonexpansive retract of $E$. Let $T_{1}, T_{2}, \ldots, T_{N}: K \rightarrow E$ be nonexpansive mappings. From arbitrary $x_{1} \in K$, define the sequence $\left\{x_{n}\right\}$ by the recursion (1.1) with for each $i=1,2, \ldots, N, \sum_{n=1}^{\infty} \gamma_{n}^{i}<\infty$. If $F:=\bigcap_{i=1}^{N} F\left(T_{i}\right) \neq \varnothing$, then for all $u, v \in F$, the limit

$$
\lim _{n \rightarrow \infty}\left\|t x_{n}+(1-t) u-v\right\|
$$

exists for all $t \in[0,1]$.

Proof. By Lemma 3.1, we have $\lim _{n \rightarrow \infty}\left\|x_{n}-x^{*}\right\|$ exists for all $x^{*} \in F$. This implies that $\left\{x_{n}\right\}$ is bounded. Observe that there exists $R>0$ such that $\left\{x_{n}\right\} \subset C:=B_{R}(0) \cap K$, and hence $C$ is a nonempty closed convex bounded subset of $E$. Let $a_{n}(t):=\left\|t x_{n}+(1-t) u-v\right\|$. Then $\lim _{n \rightarrow \infty} a_{n}(0)=\|u-v\|$, and from Lemma 3.1, $\lim _{n \rightarrow \infty} a_{n}(1)=\lim _{n \rightarrow \infty}\left\|x_{n}-v\right\|$ exists. Without loss of generality, we may assume that $\lim _{n \rightarrow \infty}\left\|x_{n}-u\right\|=r>0$ and 
$t \in(0,1)$. For any $n \geqslant 1$ and for all $i=1,2, \ldots, N$, we define $A_{n}^{i}: C \rightarrow C$ by

$$
\begin{gathered}
A_{n}^{1}:=P\left(\alpha_{n}^{1} T_{1}+\beta_{n}^{1} I+\gamma_{n}^{1} u_{n}^{1}\right), \\
A_{n}^{2}:=P\left(\alpha_{n}^{2} T_{2} A_{n}^{1}+\beta_{n}^{2} I+\gamma_{n}^{2} u_{n}^{2}\right), \\
\vdots \\
\vdots \\
A_{n}^{N}:=P\left(\alpha_{n}^{N} T_{N} A_{n}^{N-1}+\beta_{n}^{N} I+\gamma_{n}^{N} u_{n}^{N}\right) .
\end{gathered}
$$

Thus, for all $x, y \in K$, we have $\left\|A_{n}^{i} x-A_{n}^{i} y\right\| \leqslant \alpha_{n}^{i}\left\|A_{n}^{i-1} x-A_{n}^{i-1} y\right\|+\beta_{n}^{i}\|x-y\|$ for all $i=2, \ldots, N$, and $\left\|A_{n}^{1} x-A_{n}^{1} y\right\| \leqslant \alpha_{n}^{1}\|x-y\|+\beta_{n}^{1}\|x-y\|$. This implies that

$$
\left\|A_{n}^{N} x-A_{n}^{N} y\right\| \leqslant\|x-y\| .
$$

Set $S_{n, m}:=A_{n+m-1}^{N} A_{n+m-2}^{N} \cdots A_{n}^{N}, m \geqslant 1$, and $b_{n, m}:=\| S_{n, m}\left(t x_{n}+(1-t) u\right)-\left(t S_{n, m} x_{n}+\right.$ $\left.(1-t) S_{n, m} u\right) \|$. It easy to see that $A_{n}^{N} x_{n}=x_{n+1}, S_{n, m} x_{n}=x_{n+m}$, and $\left\|S_{n, m} x-S_{n, m} y\right\| \leqslant\|x-y\|$.

We show first that, for any $x^{*} \in F,\left\|S_{n, m} x^{*}-x^{*}\right\| \rightarrow 0$ uniformly for all $m \geqslant 1$ as $n \rightarrow$ $\infty$. Indeed, for any $x^{*} \in F$, we have

$$
\left\|A_{n}^{i} x^{*}-x^{*}\right\| \leqslant \alpha_{n}^{i}\left\|A_{n}^{i-1} x^{*}-x^{*}\right\|+\gamma_{n}^{i}\left\|u_{n}^{i}-x^{*}\right\|
$$

for all $i=2, \ldots, N$, and $\left\|A_{n}^{1} x^{*}-x^{*}\right\| \leqslant \gamma_{n}^{1}\left\|u_{n}^{1}-x^{*}\right\|$. Therefore

$$
\begin{aligned}
\left\|A_{n}^{N} x^{*}-x^{*}\right\| \leqslant & \sigma_{n}^{2} \gamma_{n}^{1}\left\|u_{n}^{1}-x^{*}\right\|+\sigma_{n}^{3} \gamma_{n}^{2}\left\|u_{n}^{2}-x^{*}\right\|+\cdots+\sigma_{n}^{N} \gamma_{n}^{N-1}\left\|u_{n}^{N-1}-x^{*}\right\| \\
& +\gamma_{n}^{N}\left\|u_{n}^{N}-x^{*}\right\| \leqslant M \sum_{i=1}^{N} \gamma_{n}^{i},
\end{aligned}
$$

for all $n \geqslant 1$, where $M=\max \left\{\sup _{n \geqslant 1}\left\{\left\|u_{n}^{1}-x^{*}\right\|\right\}, \ldots, \sup _{n \geqslant 1}\left\{\left\|u_{n}^{N}-x^{*}\right\|\right\}\right\}$ and $\sigma_{n}^{k}=$ $\prod_{i=k}^{N} \alpha_{n}^{i}$. Hence

$$
\begin{aligned}
\left\|S_{n, m} x^{*}-x^{*}\right\| \leqslant & \left\|A_{n+m-1}^{N} A_{n+m-2}^{N} \cdots A_{n}^{N} x^{*}-A_{n+m-1}^{N} A_{n+m-2}^{N} \cdots A_{n+1}^{N} x^{*}\right\| \\
& +\left\|A_{n+m-1}^{N} A_{n+m-2}^{N} \cdots A_{n+1}^{N} x^{*}-A_{n+m-1}^{N} A_{n+m-2}^{N} \cdots A_{n+2}^{N} x^{*}\right\| \\
& \vdots \quad \vdots \\
& +\left\|A_{n+m-1}^{N} x^{*}-x^{*}\right\| \\
\leqslant & \left\|A_{n}^{N} x^{*}-x^{*}\right\|+\left\|A_{n+1}^{N} x^{*}-x^{*}\right\|+\cdots+\left\|A_{n+m-1}^{N} x^{*}-x^{*}\right\| \\
\leqslant & M \sum_{i=1}^{N}\left(\gamma_{n}^{i}+\gamma_{n+1}^{i}+\cdots+\gamma_{n+m-1}^{i}\right) \\
\leqslant & M \sum_{i=1}^{N} \sum_{k=n}^{\infty} \gamma_{k}^{i}:=\delta_{n}^{x^{*}} .
\end{aligned}
$$


Since $\sum_{n=1}^{\infty} \gamma_{n}^{i}<\infty$, for all $i=1,2, \ldots, N$, we have $\delta_{n}^{x^{*}} \rightarrow 0$ as $n \rightarrow \infty$ and hence $\left\|S_{n, m} x^{*}-x^{*}\right\| \rightarrow 0$ as $n \rightarrow \infty$. Observe that

$$
\begin{aligned}
a_{n+m}(t)= & \left\|t S_{n, m} x_{n}+(1-t) u-v\right\| \\
\leqslant & \left\|t S_{n, m} x_{n}+(1-t) u-S_{n, m}\left(t x_{n}+(1-t) u\right)\right\| \\
& +\left\|S_{n, m}\left(t x_{n}+(1-t) u\right)-v\right\| \\
= & \left\|t S_{n, m} x_{n}+(1-t) S_{n, m} u-S_{n, m}\left(t x_{n}+(1-t) u\right)+(1-t)\left(u-S_{n, m} u\right)\right\| \\
& +\left\|S_{n, m}\left(t x_{n}+(1-t) u\right)-v\right\| \\
\leqslant & b_{n, m}+\left\|S_{n, m}\left(t x_{n}+(1-t) u\right)-v\right\|+(1-t)\left\|u-S_{n, m} u\right\| \\
\leqslant & b_{n, m}+\left\|S_{n, m}\left(t x_{n}+(1-t) u\right)-S_{n, m} v\right\|+\left\|S_{n, m} v-v\right\| \\
& +(1-t)\left\|u-S_{n, m} u\right\| \\
\leqslant & b_{n, m}+a_{n}(t)+\left\|S_{n, m} v-v\right\|+(1-t)\left\|u-S_{n, m} u\right\| \\
\leqslant & b_{n, m}+a_{n}(t)+\delta_{n}^{v}+(1-t) \delta_{n}^{u} .
\end{aligned}
$$

By using [2, Theorem 2.3], we have

$$
\begin{aligned}
b_{n, m} & \leqslant \varphi^{-1}\left(\left\|x_{n}-u\right\|-\left\|S_{n, m} x_{n}-S_{n, m} u\right\|\right) \\
& =\varphi^{-1}\left(\left\|x_{n}-u\right\|-\left\|x_{n+m}-u+u-S_{n, m} u\right\|\right) \\
& \leqslant \varphi^{-1}\left(\left\|x_{n}-u\right\|-\left(\left\|x_{n+m}-u\right\|-\left\|S_{n, m} u-u\right\|\right)\right),
\end{aligned}
$$

and so the sequence $\left\{b_{n, m}\right\}$ converges uniformly to 0 as $n \rightarrow \infty$ for all $m \geqslant 1$. Thus, fixing $n$ and letting $m \rightarrow \infty$ in (3.19), we have

$$
\limsup _{m \rightarrow \infty} a_{n+m}(t) \leqslant \varphi^{-1}\left(\left\|x_{n}-u\right\|-\left(\lim _{m \rightarrow \infty}\left\|x_{m}-u\right\|-\delta_{n}^{u}\right)\right)+a_{n}(t)+\delta_{n}^{v}+(1-t) \delta_{n}^{u}
$$

and again letting $n \rightarrow \infty$,

$$
\limsup _{n \rightarrow \infty} a_{n}(t) \leqslant \varphi^{-1}(0)+\liminf _{n \rightarrow \infty} a_{n}(t)+0+0=\liminf _{n \rightarrow \infty} a_{n}(t) .
$$

This completes the proof.

Theorem 3.9. Let E be a real uniformly convex Banach space such that its dual $E^{*}$ has the Kaded-Klee property and $K$ a nonempty closed convex subset of $E$ which is also a nonexpansive retract of $E$. Let $T_{1}, T_{2}, \ldots, T_{N}: K \rightarrow E$ be nonexpansive mappings with $F:=\bigcap_{i=1}^{N} F\left(T_{i}\right) \neq$ $\varnothing$. From arbitrary $x_{1} \in K$, define the sequence $\left\{x_{n}\right\}$ by the recursion (1.1) with for each $i=1,2, \ldots, N, \sum_{n=1}^{\infty} \gamma_{n}^{i}<\infty$ and $\alpha_{n}^{i} \in[\varepsilon, 1-\varepsilon]$ for some $\varepsilon \in(0,1)$. Then $\left\{x_{n}\right\}$ converges weakly to some fixed point of $T$.

Proof. Lemma 3.1 guarantees that $\left\{x_{n}\right\}$ is bounded. Since $E$ is reflexive, there exists a subsequence $\left\{x_{n_{j}}\right\}$ of $\left\{x_{n}\right\}$ converging weakly to some $x^{*} \in K$. By Lemma 3.2, we have 
$\lim _{j \rightarrow \infty}\left\|x_{n_{j}}-T_{i} x_{n_{j}}\right\|=0$ for all $i=1,2, \ldots, N$. Now Lemma 2.4 guarantees that $I-T_{i}$ is demiclosed at zero for all $i=1,2, \ldots, N$. This implies that $T_{i} x^{*}=x^{*}$ for all $i=1,2, \ldots, N$, hence this means that $x^{*} \in F$. It remains to show that $\left\{x_{n}\right\}$ converges weakly to $x^{*}$. Suppose $\left\{x_{n_{i}}\right\}$ is another subsequence of $\left\{x_{n}\right\}$ converging weakly to some $y^{*}$. Then $y^{*} \in K$ and so $x^{*}, y^{*} \in \omega_{w}\left(x_{n}\right) \cap F$. By Lemma 3.8, the limit

$$
\lim _{n \rightarrow \infty}\left\|t x_{n}+(1-t) x^{*}-y^{*}\right\|
$$

exists for all $t \in[0,1]$. By Lemma 2.3 we have $x^{*}=y^{*}$. As a result, $\omega_{w}\left(x_{n}\right) \cap F$ is a singleton, and so $\left\{x_{n}\right\}$ converges weakly to some fixed point of $T$.

Corollary 3.10 (see [12, Theorem 3.5]). Let E be a real uniformly convex Banach space such that its dual $E^{*}$ has the Kadec-Klee property and $K$ a nonempty closed convex subset of $E$ which is also a nonexpansive retract of $E$. Let $T: K \rightarrow E$ be a nonexpansive mapping with $F(T) \neq \varnothing$. Let $\left\{\alpha_{n}\right\}$ and $\left\{\beta_{n}\right\}$ be sequences in $[\varepsilon, 1-\varepsilon]$ for some $\varepsilon \in(0,1)$. From arbitrary $x_{1} \in K$, define the sequence $\left\{x_{n}\right\}$ by the recursion (1.2). Then $\left\{x_{n}\right\}$ converges weakly to some fixed point of $T$.

\section{Acknowledgments}

The authors would like to thank the Faculty of Science, Naresuan University, and the Thailand Research Fund for financial support. Moreover, the authors thank the referees for their valuable suggestions to improve this manuscript. This work is supported by Thailand Research Fund. K. Ungchittrakool is also supported by the Royal Golden Jubilee Program under Grant PHD/0086/2547, Thailand.

\section{References}

[1] F. E. Browder, Semicontractive and semiaccretive nonlinear mappings in Banach spaces, Bulletin of the American Mathematical Society 74 (1968), 660-665.

[2] J. García Falset, W. Kaczor, T. Kuczumow, and S. Reich, Weak convergence theorems for asymptotically nonexpansive mappings and semigroups, Nonlinear Analysis 43 (2001), no. 3, 377-401.

[3] S. Ishikawa, Fixed points by a new iteration method, Proceedings of the American Mathematical Society 44 (1974), no. 1, 147-150.

[4] J. S. Jung and S. S. Kim, Strong convergence theorems for nonexpansive nonself-mappings in Banach spaces, Nonlinear Analysis 33 (1998), no. 3, 321-329.

[5] W. Kaczor, Weak convergence of almost orbits of asymptotically nonexpansive commutative semigroups, Journal of Mathematical Analysis and Applications 272 (2002), no. 2, 565-574.

[6] S. H. Khan and H. Fukhar-ud-din, Weak and strong convergence of a scheme with errors for two nonexpansive mappings, Nonlinear Analysis 61 (2005), no. 8, 1295-1301.

[7] W. R. Mann, Mean value methods in iteration, Proceedings of the American Mathematical Society 4 (1953), no. 3, 506-510.

[8] S.-Y. Matsushita and D. Kuroiwa, Strong convergence of averaging iterations of nonexpansive nonself-mappings, Journal of Mathematical Analysis and Applications 294 (2004), no. 1, 206214.

[9] Z. Opial, Weak convergence of the sequence of successive approximations for nonexpansive mappings, Bulletin of the American Mathematical Society 73 (1967), 591-597.

[10] B. E. Rhoades, Fixed point iterations for certain nonlinear mappings, Journal of Mathematical Analysis and Applications 183 (1994), no. 1, 118-120. 


\section{Weak and strong convergence}

[11] J. Schu, Iterative construction of fixed points of asymptotically nonexpansive mappings, Journal of Mathematical Analysis and Applications 158 (1991), no. 2, 407-413.

[12] N. Shahzad, Approximating fixed points of non-self nonexpansive mappings in Banach spaces, Nonlinear Analysis 61 (2005), no. 6, 1031-1039.

[13] W. Takahashi and G.-E. Kim, Strong convergence of approximants to fixed points of nonexpansive nonself-mappings in Banach spaces, Nonlinear Analysis 32 (1998), no. 3, 447-454.

[14] W. Takahashi and T. Tamura, Convergence theorems for a pair of nonexpansive mappings, Journal of Convex Analysis 5 (1998), no. 1, 45-56.

[15] K.-K. Tan and $\mathrm{H}$. K. Xu, Approximating fixed points of nonexpansive mappings by the Ishikawa iteration process, Journal of Mathematical Analysis and Applications 178 (1993), no. 2, 301-308.

[16] _ Fixed point iteration processes for asymptotically nonexpansive mappings, Proceedings of the American Mathematical Society 122 (1994), no. 3, 733-739.

[17] H. K. Xu, Inequalities in Banach spaces with applications, Nonlinear Analysis 16 (1991), no. 12, $1127-1138$.

[18] Y. Xu, Ishikawa and Mann iterative processes with errors for nonlinear strongly accretive operator equations, Journal of Mathematical Analysis and Applications 224 (1998), no. 1, 91-101.

[19] H. K. Xu and X. M. Yin, Strong convergence theorems for nonexpansive non-self-mappings, Nonlinear Analysis 24 (1995), no. 2, 223-228.

S. Plubtieng: Department of Mathematics, Faculty of Science, Naresuan University,

Phitsanulok 65000, Thailand

E-mail address: somyotp@nu.ac.th

K. Ungchittrakool: Department of Mathematics, Faculty of Science, Naresuan University, Phitsanulok 65000, Thailand

E-mail address: g47060127@nu.ac.th 